\title{
Hexokinase 2 is dispensable for T cell-dependent immunity
}

\author{
Manan M. Mehta', Samuel E. Weinberg ${ }^{1}$, Elizabeth M. Steinert ${ }^{1}$, Krishan Chhiba' ${ }^{1}$, Carlos Alberto Martinez², \\ Peng Gao ${ }^{3}$, Harris R. Perlman', Paul Bryce ${ }^{1}$, Nissim Hay ${ }^{4}$ and Navdeep S. Chandel ${ }^{\text {** (I) }}$
}

\begin{abstract}
Background: T cells and cancer cells utilize glycolysis for proliferation. The hexokinase (1-4) family of enzymes catalyze the first step of glycolysis. Hexokinase 2 (HK2) is one of the most highly upregulated metabolic enzymes in both cancer and activated T cells. HK2 is required for the development and/or growth of cancer in several cancer models, but the necessity of HK2 in T cells is not fully understood. The clinical applicability of HK2 inhibition in cancer may be significantly limited by any potential negative effects of HK2 inhibition on T cells. Therefore, we investigated the necessity of HK2 for T cell function. In order to identify additional therapeutic cancer targets, we performed RNA-seq to compare in vivo proliferating $T$ cells to $T$ cell leukemia.

Methods: HK2 was genetically ablated in mouse T cells using a floxed HK2 allele crossed to CD4-Cre. CD4+ and CD8+ cells from mice were characterized metabolically and tested in vitro. T cell function in vivo was tested in a mouse model of colitis, Th2-mediated lung inflammation, and viral infection. Treg function was tested by crossing Hk2-floxed mice to FoxP3-Cre mice. Hematopoietic function was tested by deleting HK2 from bone marrow with Vav1-iCre. RNA-seq was used to compare T cells proliferating in response to virus with primary T-ALL leukemia induced with mutant Notch1 expression.
\end{abstract}

Results: We unexpectedly report that HK2 is largely dispensable for in vitro T cell activation, proliferation, and differentiation. Loss of HK2 does not impair in vivo viral immunity and causes only a small impairment in the development of pathological inflammation. HK2 is not required for Treg function or hematopoiesis in vivo. One hundred sixty-seven metabolic genes were identified as being differentially expressed between $T$ cells and leukemia.

Conclusions: HK2 is a highly upregulated enzyme in cancer and in T cells. The requirement for HK2 in various cancer models has been described previously. Our finding that T cells are able to withstand the loss of HK2 indicates that HK2 may be a promising candidate for cancer therapy. Furthermore, we identify several other potential metabolic targets in T-ALL leukemia that could spare T cell function.

Keywords: Hexokinase 2, T cells, Tregs, Leukemia, Colitis, LCMV

\section{Background}

$\mathrm{T}$ lymphocytes in the immune system and neoplastic cells in cancer are both highly proliferative cell types, relying on highly active metabolic pathways $[1,2]$. T cells typically divide and expand in response to a specific antigen, while cancer cells exhibit uncontrolled proliferation. Nonetheless, similar molecular mechanisms underlie the ability of these different cell types to proliferate.

\footnotetext{
* Correspondence: nav@northwestern.edu

'Department of Medicine, Northwestern University Feinberg School of Medicine,

McGaw Pavilion, Rm. M-334, 240 East Huron Street, Chicago, IL 60611, USA

Full list of author information is available at the end of the article
}

Indeed, one of the major side effects of chemotherapies which target proliferating cancer cells is immunosuppression. One common mechanism utilized by both cancer and activated $\mathrm{T}$ cells to support their proliferation is an upregulation of glycolysis to provide intermediates for macromolecule synthesis $[3,4]$.

Glucose metabolism is essential for both normal $\mathrm{T}$ cell-mediated immunity and pathological $\mathrm{T}$ cell-mediated inflammation [5-11]. Studies have similarly shown that glucose metabolism is required for tumor growth in a variety of cancer models [12]. Furthermore, in the tumor microenvironment, evidence indicates that $\mathrm{T}$ cells and

(c) The Author(s). 2018 Open Access This article is distributed under the terms of the Creative Commons Attribution 4.0 International License (http://creativecommons.org/licenses/by/4.0/), which permits unrestricted use, distribution, and 
cancer cells may compete for glucose and that cancer cells may upregulate glycolysis in part to deprive T cells of glucose as a means to evade and suppress the immune system $[13,14]$. These studies have led to interest in using anti-glycolytic agents for cancer therapy. Clearly, glucose metabolism is essential for both cancer and $\mathrm{T}$ cells. However, it is not clear if glucose is utilized differently in cancer and $\mathrm{T}$ cells and thus if glucose metabolism is a viable target for cancer therapy. Given that $\mathrm{T}$ cells also rely heavily on glucose, it is possible that anti-glycolytic therapy could lead to immunosuppression, leaving patients susceptible to infections. Furthermore, such immunosuppression would work at cross purposes with emerging cancer therapies such as check point blockade which rely on $\mathrm{T}$ cells. Therefore, any potential glycolytic target for cancer therapy should ideally spare T cell function.

One possible target for anti-glycolytic cancer therapy is hexokinase (HK), first committed step of glycolysis. HK can be expressed as four different isoforms, with HK1 being a somewhat ubiquitous isoform and HK2 existing as a more selectively regulated isoform [15]. Cancer cells highly upregulate HK2 compared to their normal tissue of origin and it is required for tumorigenesis in a variety of mouse cancer models, including breast and lung cancer and T cell leukemia [16-19]. However, HK2 is also highly upregulated in activated $\mathrm{T}$ cells [20, 21]. Furthermore, pan-hexokinase inhibition with 2-deoxyglucose (2DG) causes impaired differentiation of the inflammatory Th17 CD4+ T cell lineage and a shift from effector to memory cells in CD8+ T cells [20, 22, 23]. Presently, it is unknown whether the loss of HK2 would impair T cell-mediated inflammation and immunity.

In this study, we report that HK2 is largely dispensable in $\mathrm{T}$ cells for viral immunity and inflammation in several mouse models. Collectively, our data indicate that targeting HK2 may be a promising avenue for further research in cancer therapy, as it may spare $\mathrm{T}$ cell function while inhibiting cancer growth.

\section{Methods}

\section{Mouse models}

$\mathrm{HK} 2^{\mathrm{fl} / \mathrm{fl}}$ mice were previously generated [16]. HK2 WT, floxed (Fl), and null alleles were genotyped using the following primers: HK2-F (CCCCTTCGCTTGCCATTAC), HK2-R (TGTCTTGGCTCAGATGTGAC), and HK2-null (AACCACGACGCCCAATGATTTAG). CD4-Cre, Vav-iCre, FoxP3-YFP-Cre, CD45.1, and IL-10 ${ }^{-1-}$ mice were obtained from the Jackson Labs. Except where noted otherwise, approximately 2-4-month old adult mice of both sexes were used for experiments. Animals were not randomized to experimental groups, but were age-matched, sex-matched, and littermates where possible. No animals were excluded from analysis. Investigators were blinded to mouse genotypes for histological scoring but not blinded otherwise.
All animals were housed and bred in the Northwestern animal vivarium, and procedures were approved by the Institutional Animal Care and Use Committee (IACUC) at Northwestern University.

\section{T cell isolation and in vitro culture}

Spleens from approximately 2-4-month old mice were mechanically disrupted through a $70 \mathrm{uM}$ strainer in $2 \%$ FBS/PBS to yield splenocytes. CD4+ T cells and CD8+ T cells were isolated from splenocytes using a negative selection magnetic bead kit according to manufacturer's protocol (StemCell). Resulting T cells were activated 1:1 with CD3/CD28-coated beads (Gibco) and $20 \mathrm{ng} / \mathrm{mL}$ IL-2 (PeproTech) for 24-72 h in RPMI 1640 media, 10\% fetal bovine serum (FBS), $10 \mathrm{mM}$ HEPES, $2 \mathrm{mM}$ glutamax, and $50 \mathrm{uM}$ beta-mercaptoethanol with antibiotic/ antimycotic. Oxygen consumption and extracellular acidification rate were measured as previously descried using a Seahorse XF96 analyzer (Agilent) [24]. For proliferation experiments, cells were stained with CFSE prior to activation according to manufacturer's protocol (Thermo Fisher). For low glucose experiments, dialysed fetal bovine serum was used and glucose was added as indicated. For experiments requiring conditions biased for certain lineages, activation was altered as follows. Cells were activated with 5:1 irradiated splenocytes $(3000 \mathrm{rad})$ and $2.5 \mu \mathrm{g} / \mathrm{mL}$ soluble anti-CD3 (eBiosciences). Cytokines and blocking antibodies were added to promote Th1 $(10 \mathrm{ng} / \mathrm{mL}$ IL-12, $10 \mu \mathrm{g} / \mathrm{mL}$ anti-IL-4), Th2 (300 ng/mL IL-4, $10 \mu \mathrm{g} / \mathrm{mL}$ anti-IL-12, $10 \mu \mathrm{g} / \mathrm{mL}$ anti-IFN $\gamma$ ), Th17 (2 ng/mL TGFb, $20 \mathrm{ng} / \mathrm{mL}$ IL-6), and Treg (2 ng/mL TGFb) differentiation. Th0 consisted of base media without any additional cytokines or blocking antibodies. IL-12 was obtained from PeproTech, anti-IL-4 and anti-IFNY from BioLegend, IL-6 and anti-IL-12 from eBiosciences, and IL-4/TGFb from R\&D. Cells split 1:2 after 3 days and supplemented with additional media and IL-2 for 2 days before analysis.

\section{Glucose assays}

HK activity and 2-DG uptake were measured on activated $\mathrm{CD} 4+$ and CD8+ T cells by commercially available kits (BioVision and Promega, respectively) according to manufacturer's instructions. HK lysates were flash frozen in liquid nitrogen prior to storage at $-80^{\circ} \mathrm{C}$.

\section{Flow cytometry and sorting}

Single-cell suspensions were resuspended in 2\% FBS/ PBS for surface staining, and the following antibodies were used as indicated (clone numbers in parenthesis): anti-CD4 (GK1.5, RM4-5), CD8 (53-6.7), CD45.2 (104), CD25 (PC61.5), CD69 (H1-2F3), CD44 (IM7), CD62L (MEL14), F4/80 (BM8), CD11c (N418), B220 (RA3-6B2), CD11b (M1/70), Gr-1 (RB6-8C5), NK1.1 (PK136), 
TER-119 (TER-119), and CD19 (1D3). Viability was determined by exclusion of nucleic dye, either propidium iodide (Thermo Fisher), LIVE/DEAD Fixable Blue stain (Thermo Fisher), or Ghost Dye Red 780 (Tonbo). For intracellular cytokine staining, cells were pretreated with $50 \mathrm{ng} / \mathrm{mL}$ PMA (Sigma), $1 \mu \mathrm{g} / \mathrm{mL}$ ionomycin (Sigma), and protein transport inhibitor cocktail (eBiosciences) for approximately $4 \mathrm{~h}$ (except in case of gp33 stimulation, which is performed as described below). For intracellular cytokines and transcriptions factors, cells were fixed with a Cell Fixation/Permeabilization Kit (BD) or FoxP3/Transcription Factor Staining Kit (eBiosciences) and stained with the following antibodies as indicated: anti-Tbet (O4-46), GATA3 (TWAJ), RORyt (B2D), FoxP3 (FJK-16 s), IFNY (XMG1.2), IL-4(11B11), and IL-17a (17B7). Absolute counts were obtained by running samples with a known concentration of beads (Spherotech) or by counting cells on a Cellometer (Nexcelom). gp33 monomer (D(b)/LCMV.GP33.KAVYN FATM) was obtained from the NIH Tetramer Core Facility and tetramerized according to their provided protocol. All samples were run on LSR Fortessa or FACSymphony flow cytometers (BD) and data was analyzed using FlowJo software. Cells were sorted using the FASC Aria II (BD). Sorted cells were reanalyzed afterwards to ensure approximately $90 \%$ purity.

\section{Protein extraction and western blot}

Cells pellets were stored at $-80{ }^{\circ} \mathrm{C}$ until resuspension and lysis. Protein content was quantified by BCA assay. Four to twenty percent or Any $\mathrm{kD}$ polyacrylamide gels (Bio-Rad) were used to separate lysate proteins. Protein was then transferred to nitrocellulose membranes using Trans-Blot Turbo (Bio-Rad). Membranes were blocked using 5\% milk/TBS for $1 \mathrm{~h}$, washed with TBST, and then incubated in 5\% BSA/TBST with anti-HK2 (Santa Cruz, product \#6521, diluted approximately 1:500) and anti- $\beta$-actin (Sigma, catalog no. T9026/A2066; diluted 1:1000) or anti-GAPDH (Santa Cruz, clone C65, diluted 1:1000) overnight. HK1 and HK3 blotting performed similarly with antibodies from Cell Signaling (clone C35C4) and Abcam (catalog no. ab126217), respectively. Membranes were washed with TBST and incubated in appropriate secondary antibodies-donkey anti-goat or goat anti-rabbit IRDye $800 \mathrm{CW}$ (LI-COR, diluted 1:5000-1:10000 in 5\% milk/TBST) and donkey anti-mouse or anti-rabbit IRDye 680RD (LI-COR, diluted $1: 10,000$ in $5 \%$ milk/TBST)-for $1 \mathrm{~h}$ at room temperature. Membranes were imaged using the Odyssey Fc Analyzer (LI-COR).

\section{RNA isolation and real-time PCR}

RNA was isolated using standard lab protocols. cDNA was generated using M-MLV Reverse Transcriptase
(Invitrogen). Real-time PCR was performed on the CFX384 Real-Time System (Bio-Rad) using iQ SYBR Green Supermix (Bio-Rad) and $200 \mathrm{nM}$ primers. Alternatively, low quantity RNA samples were analyzed using a one-step protocol (Tonbo CYBRFast 1-Step RT-qPCR Lo-Rox). Primers used as follows: HK2-F (GTGTGCTCCGAGTAAGGGTG), HK2-R (CAGGCAT TCGGCAATGTGG), HK1-F (AACGGCCTCCGTCA AGATG), HK1-R (GCCGAGATCCAGTGCAATG), HK 3-F (TGCTGCCCACATACGTGAG), HK3-R (GCCTG TCAGTGTTACCCACAA), Hmgcs2-F (GAAGAGAGC GATGCAGGAAAC), Hmgcs2-R (GTCCACATATTGG GCTGGAAA), Chdh-F (TTCGGCTGGATGGACATGA C), Chdh-R (CTGCTTACAAGTGTCTGGACC), Ldhb-F (CATTGCGTCCGTTGCAGATG), Ldhb-R (GGAGGAA CAAGCTCCCGTG), Prodh-F (GCACCACGAGCAGT TGTTC), Prodh-R (CTTTGTTGTGCCGGATCAGAG), Fbp1-F (CACCGCGATCAAAGCCATCT), Fbp1-R (AGG TAGCGTAGGACGACTTCA), b-Actin-F (CTAAGGCC AACCGTGAAAAG), b-Actin-R RPL-19-F (GAAGGTCA AAGGGAATGTGTTCAA), and RPL-19-R (TTTCGTGC TTCCTTGGTCTTAGA). Data was analyzed using $\triangle \mathrm{CT}$ method by comparing genes of interest to the geometric mean of indicated housekeeping genes.

\section{Colitis model and histology}

IL $10^{-/-}$mice were allowed to spontaneously develop colitis, typically between 8 and 14 weeks of age. Mice were euthanized if they lost greater than $20 \%$ of their body weight or if they developed rectal prolapse. IL- $10^{+/+}$littermates were euthanized at approximately $13-15$ weeks as controls. Colon, spleen, and mesenteric lymph nodes from euthanized mice were collected. Colons were fixed for 2 days in 10\% neutral buffered formalin for $H \& E$ staining or immediately digested to isolate lamina propria cells as previously described [25]. Lymph nodes were mechanically disrupted in RPMI on scored plates to yield lymphocytes. Lymphocytes were analyzed by flow cytometry as described above. Fixed tissues were paraffin-embedded, sectioned, mounted, and stained with $\mathrm{H} \& \mathrm{E}$ by the Northwestern mouse histology core. Scoring was performed blinded according to established criteria [26].

\section{OVA immunization and airway inflammation}

OVA immunization and challenge was performed as previously described [27]. Six to eight-week old mice were sensitized to OVA by treating with intraperitoneal $10 \mathrm{mg}$ OVA (Grade VI, Sigma-Aldrich) in alum (3 $\mathrm{mg}$ ) or equal volume PBS/alum on days 0 and 14. On days 21-23, mice were challenged daily with $1 \%$ OVA/PBS aerosolized by nebulizer. Mice were euthanized on day 24, trachea was cannulated, and lungs were flushed with PBS $+10 \%$ FCS +1 mM EDTA. Resulting BAL fluid was counted for total nucleated cell count, cells were cytospun onto slides, and supernatants 
were used for ELISA cytokine quantification. Differential counts were performed after staining slides with DiffQuik. Lung tissue was homogenized for RNA isolation (Qiagen) and analyzed by TaqMan probes according to manufacturer's protocol (Thermo Scientific, probe numbers mm00445259_m1, mm00439646_m1, mm00434204_m1, mm01168134_m1, and mm00439618_m1). Remaining lung tissue was fixed and H\&E stained as described above.

\section{LCMV infection, CD8+ T cell restimulation, and plaque assay}

Adult mice were infected with 200,000 pfu LCMV-Armstrong intraperitoneally. Peripheral blood was acquired by retro-orbital bleeds on indicated days. Red blood cells were lysed in RBC lysis buffer (eBiosciences) and blood was analyzed by flow cytometry as described above. Mice were euthanized after 60 days post infection (DPI) and splenocytes were isolated as described above. One million splenocytes per sample were stimulated with $30 \mathrm{ng} / \mathrm{mL}$ exogenous gp $_{33-41}$ peptide (KAVYNFATM, GenScript) for approximately $5 \mathrm{~h}$ in the presence of GolgiPlug (BD), after which surface staining, tetramer staining, and intracellular cytokine staining were performed as described above. Alternatively, mice were euthanized at three and eight DPI, and liver and spleens were removed. Eight DPI spleens were sorted for CD8+ T cells which were used for RNA-seq and metabolomics as described below. Liver was homogenized and used to determine viral load by plaque assay as described in detail by others [28]. Briefly, serial dilutions of liver homogenate were added to a monolayer of Vero cells and overlayed with $0.5 \%$ agarose for 5 days, stained with neutral red for 1 day, and PFUs were counted.

\section{Bone marrow isolation and leukemic transformation}

Peripheral blood counts were obtained by retro-orbital bleeds of mice and analysis on a HEMAVET (Drew). Bone marrow from adult mice was harvested by dissecting pelvis, femur, and tibia. Bones were centrifuged for $5 \mathrm{~min}$ at $5000 \mathrm{rcf}$ in an 18G-punctured $500 \mathrm{uL}$ tube nested in a $1.5 \mathrm{~mL}$ tube. Platinum-E cells were used to create Notch1-DE retrovirus by treating cells with MIGR1-Notch1-DE plasmid and using the jetPRIME manufacturer's protocol (plasmid and cell line gifted by Panagiotis Ntziachristos). Bone marrow was used with a CD117+ positive selection magnetic bead isolation kit (StemCell) to yield CD117+ cells. CD117+ cells were grown in Opti-MEM (Thermo Fisher) supplemented with $10 \mathrm{ng} / \mathrm{mL}$ IL-3 (PeproTech), $10 \mathrm{ng} / \mathrm{mL} \quad$ IL-7 (PeproTech), 50 ng/mL SCF (PeproTech), 50 ng/mL Flt3L (PeproTech), and $20 \mathrm{ng} / \mathrm{mL}$ IL-6 (eBiosciences). Cells were infected with Notch1-DE retrovirus by adding virus to cells, centrifugation at $2500 \mathrm{rpm}$ at $25{ }^{\circ} \mathrm{C}$ for $90 \mathrm{~min}$, incubation at $37^{\circ} \mathrm{C}$ for $4 \mathrm{~h}$, and removal of virus replacing with previous media. Infection was repeated on the next day, after which cells were allowed to rest for 2 days. Seventy-five thousand lineage (CD4, CD8a, B220, CD11b, Gr-1, NK1.1, Ter-119)-negative, GFP+ cells were sorted and mixed with 750,000 bone marrow cells from CD45.1 mice resuspended in PBS. Cells were adoptively transferred into lethally irradiated (1000 rad) CD45.1 mice by retro-orbital injection. Mice were monitored over 6 weeks by analyzing peripheral blood with flow cytometry for CD45.2 + GFP + CD4 + CD8+ leukemic cells to ensure development of leukemia. Splenocytes were sorted for leukemic cells and analyzed by RNA-seq (described below).

\section{RNA-seq data acquisition and analysis}

RNA quality and quantity were measured using Agilent 4200 TapeStation using High Sensitivity RNA ScreenTape System (Agilent Technologies). SMART-Seq v4 Ultra Low Input RNA Kit (Takara Bio USA, Inc.) was used to generate full-length cDNA, and NextEra XT DNA sample preparation kit (Illumina Inc.) was used to prepare the final library. An Illumina NextSeq 500 (Illumina Inc.) was used to sequence library with single-end sequencing $(1 \times 75 \mathrm{cy}$ cles) using NextSeq 500 High Output reagent kit (Illumina Inc.). FASTQ reads were trimmed using Trimmomatic to remove end nucleotides with a PHRED score less than 30 and requiring a minimum length of $20 \mathrm{bp}$. Reads were then aligned to the mm10 genome using TopHat version 2.1.0 using the following options: --no-novel-juncs, --read-mismatches 2, --read-edit-dist 2, --max-multihits 20, and --library-type fr-unstranded. The generated bam files were then used to count the reads only at the exons of genes using htseq-count with the following parameters: - $\mathrm{q}-\mathrm{m}$ intersection-nonempty $-\mathrm{s}$ no - $\mathrm{t}$ exon. The $\mathrm{R}$ package edge $\mathrm{R}$ was used to determine significance of differentially expressed genes, only genes with $>2$-fold change in expression and adjusted $p$ value $<0.01$ were considered significant and included in heatmap. Genes were filtered for involvement in metabolism based on the KEGG mmu01100 genes. Broad Institute GSEA software was used to perform a pre-ranked GSEA analysis using 3000 permutations and the Hallmark pathway database.

\section{Metabolomics}

Cell pellets were flash frozen in liquid nitrogen and stored at $-80{ }^{\circ} \mathrm{C}$ until metabolite extraction. Samples were thawed and pellets were resuspended in ice cold $80 \%$ methanol, freeze-thawed three times, and centrifuged at $18,000 \mathrm{rcf}$ for $10 \mathrm{~min}$ at $4{ }^{\circ} \mathrm{C}$. Supernatants were transferred to new tubes, dried, resuspended, and volume-adjusted so 500,000 cells' worth of metabolites were analyzed for targeted metabolomics. Samples were analyzed by High-Performance Liquid Chromatography and High-Resolution Mass Spectrometry and Tandem Mass Spectrometry (HPLC-MS/MS). Specifically, system consisted of a Thermo $Q$ Exactive in line with an 
electrospray source and an Ultimate3000 (Thermo) series HPLC consisting of a binary pump, degasser, and auto-sampler outfitted with a Xbridge Amide column (Waters; dimensions of $4.6 \mathrm{~mm} \times 100 \mathrm{~mm}$ and a $3.5-\mu \mathrm{m}$ particle size). The mobile phase A contained 95\% (vol/ vol) water, $5 \%(\mathrm{vol} / \mathrm{vol})$ acetonitrile, $20 \mathrm{mM}$ ammonium hydroxide, $20 \mathrm{mM}$ ammonium acetate, $\mathrm{pH}=9.0$; and $\mathrm{B}$ was $100 \%$ acetonitrile. The gradient was: $0-1 \mathrm{~min}, 15 \%$ A; 18.5 min, $76 \%$ A; $18.5-20.4 \mathrm{~min}, 24 \%$ A; 20.4$20.5 \mathrm{~min}, 15 \% \mathrm{~A}$; and $20.5-28 \mathrm{~min}, 15 \%$ A with a flow rate of $400 \mu \mathrm{L} / \mathrm{min}$. The capillary of the ESI source was set to $275^{\circ} \mathrm{C}$, with sheath gas at 45 arbitrary units, auxiliary gas at 5 arbitrary units, and the spray voltage at $4.0 \mathrm{kV}$. In positive/negative polarity switching mode, an $\mathrm{m} / \mathrm{z}$ scan range from 70 to 850 was chosen and MS1 data was collected at a resolution of 70,000. The automatic gain control (AGC) target was set at $1 \times 106$ and the maximum injection time was $200 \mathrm{~ms}$. The top five precursor ions were subsequently fragmented, in a data-dependent manner, using the higher energy collisional dissociation (HCD) cell set to $30 \%$ normalized collision energy in MS2 at a resolution power of 17,500. Data acquisition and peak extraction/integration were carried out by Xcalibur 4.0 software and TraceFinder 2.1 software, respectively (Thermo Fisher). Low quality peaks were omitted from analysis. Resulting ion counts were normalized to total ion counts per sample and analyzed using multiple $t$ tests with a Benjamini-Kreiger false discovery rate of $10 \%$.

\section{Statistical analyses}

Data were analyzed statistically using Prism 7 (GraphPad), except RNA-seq data which was analyzed as described above. Statistical tests, error bar representations, $p$ values, biological replicates, sample sizes, and independent experiments are specified in figure legends. Appropriate statistical tests were selected for each comparison based on type of data and assuming normality. Adjustments for multiple comparisons were performed when appropriate and are described in figure legends. Representative images of gels and histology are from at least three biological replicates. No outliers were removed from data analysis.

\section{Results}

\section{HK2 is dispensable for T cells in vitro}

To assess the necessity of HK2 for basic T cell function in vitro, we generated mice that have a conditional deletion of the Hk2 gene in $\mathrm{T}$ cells by crossing CD4-Cre mice with mice harboring a floxed $H k 2$ allele $\left(H k 2^{\mathrm{t} / \mathrm{fl}}\right)$. Resulting

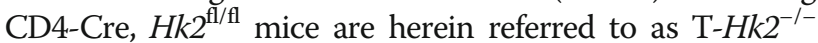
mice. Spleens from the mice were removed and CD4+ and CD8+ cells were isolated and activated in vitro. As has been observed previously, CD4+ and CD8+ WT T cells highly upregulated $H k 2$ transcript upon activation [20], but T cells from $\mathrm{T}-H k 2^{-/-}$mice had no increase in HK2 mRNA or protein upon activation (Fig. 1a, b, Additional file 1: Figure S1A-B, Additional file 2: Figure S2A). Importantly, there was no compensatory increase in HK1 or HK3 mRNA or protein in $\mathrm{CD} 4+$ or $\mathrm{CD} 8+$ cells from $\mathrm{T}-\mathrm{Hk} 2^{-/-}$mice (Fig. 1a, b, Additional file 1: Figure S1A-B). Surprisingly, total HK activity measured in activated $\mathrm{CD} 4+$ and $\mathrm{CD} 8+$ cells was not statistically different between WT and T- $H k 2^{-/-}$mice. (Fig. 1c). Glucose uptake capacity was measured by the amount of 2-DG taken up by cells. 2-DG uptake was unaffected by HK2 deficiency in CD4+ and CD8+ T cells (Fig. 1d). Basal and maximal extracellular acidification rate (ECAR) is a surrogate measure of lactate production and glycolytic activity. ECAR was similar between activated T cells from WT and T- $H k 2^{-1-}$ mice (Fig. 1e). Oxygen consumption rate was also unchanged in activated CD4+ and CD8+ T cells (Fig. 1f). Overall, our data show that HK2 deficiency causes only small changes in T cell glucose usage.

In order to determine if HK2-deficient T cells had any functional deficits in vitro, we measured their ability to activate and proliferate after stimulation. Surprisingly, HK2-deficient CD4+ and CD8+ T cells had no decrease in expression of the activation markers CD25 or CD69 after $24 \mathrm{~h}$ (Fig. 2a, Additional file 3: Figure S3A). CD4+ $\mathrm{T}$ cells had no decrease in proliferation after $72 \mathrm{~h}$ as measured by CFSE staining, while CD8+ T cells consistently showed a small but insignificant deficit (Fig. 2b). Together, these data indicate that HK2 is not required for $\mathrm{T}$ cell viability, activation, or proliferation in vitro.

Another important function of T cells is their ability to differentiate into specialized subsets. We tested the ability of CD4+ cells to differentiate in vitro by skewing them towards Th1, Th2, Th17, and Treg lineages. No major impairment was observed between WT- and HK2-deficient $\mathrm{T}$ cells in any of these lineages as determined by viability and expression of lineage defining transcription factors (Fig. 3a-e, Addtional file 3: Figure S3C). We also compared the steady state levels of different cell types in the spleens of WT and T- $H k 2^{-1-}$ mice. We observed no differences in percent of $\mathrm{CD} 4+, \mathrm{CD} 8+$, naïve $\left(\mathrm{CD} 62 \mathrm{~L}^{\mathrm{hi}} \mathrm{CD} 44^{\text {lo }}\right)$, memory $\left(\mathrm{CD} 44^{\mathrm{hi}}\right)$, Th1, Th2, Th17, or Treg cells in T- $H k 2^{-/-}$mice (Fig. 3f-h, Additional file 4: Figure S4F, Additional file 3: Figure S3B). Furthermore, there were no observed differences in other immune cell numbers (Additional file 4: Figure S4G). Together, these data demonstrate that T-Hk2-/mice do not have an impairment in $\mathrm{T}$ cell differentiation in vitro or at steady state in vivo.

The unexpected dispensability for HK2 in vitro lead us to hypothesize that perhaps $\mathrm{HK} 2$ is upregulated in T cells to maintain glycolysis during conditions of low glucose availability. To this end, we activated CD4+ T cells in vitro under various glucose concentrations, from 


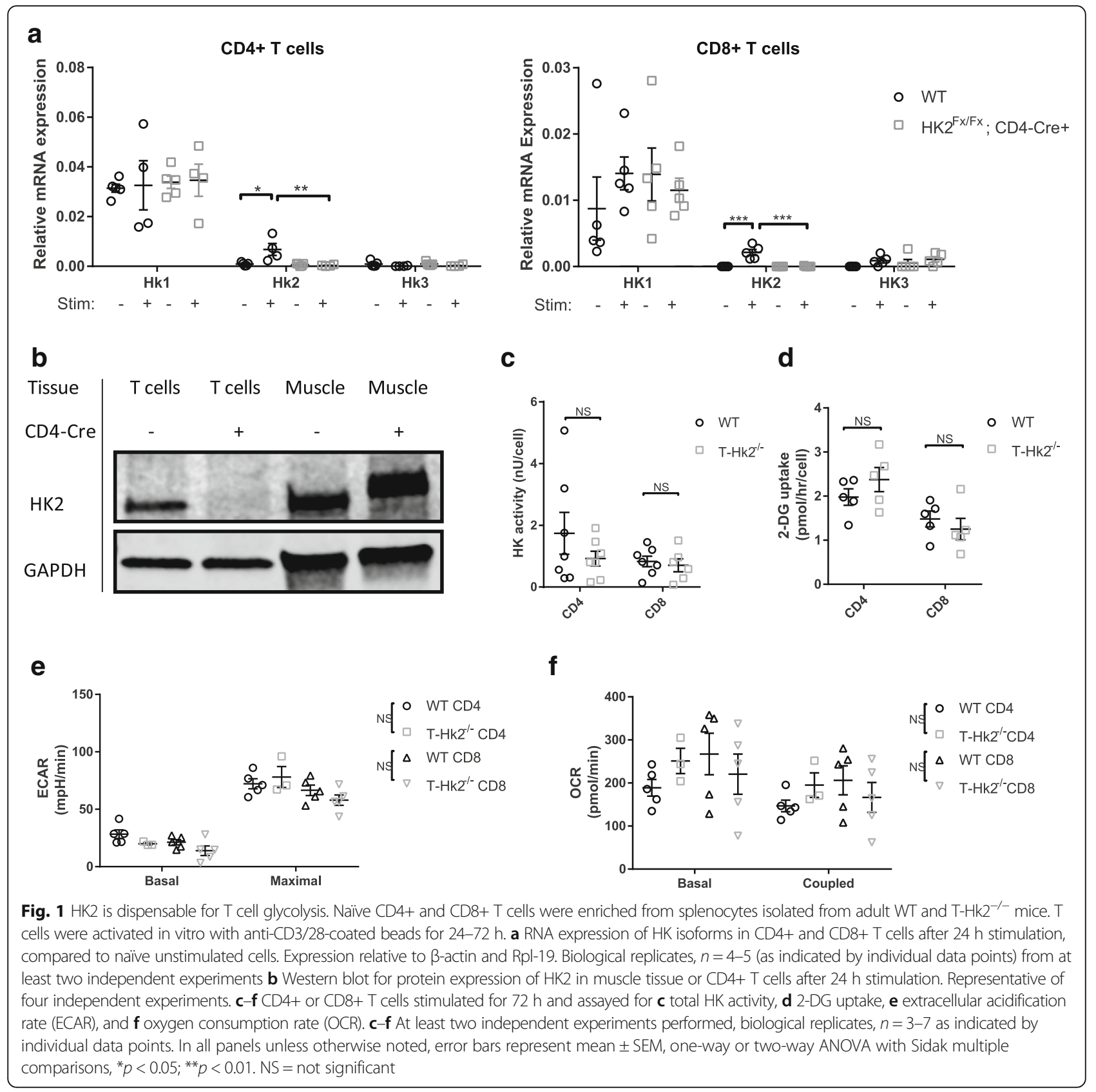

$5.5 \mathrm{mM}$ (physiological blood glucose) to $0 \mathrm{mM}$. Previous work has shown that activation and viability at $24 \mathrm{~h}$ should not be affected by low concentrations of glucose, but the complete absence of glucose will cause $\mathrm{T}$ cell death that can be rescued by pyruvate $[24,29]$. Consistent with previous findings, we observed a sharp decline in viability and activation in WT T cells between $0.1 \mathrm{mM}$ glucose and $0 \mathrm{mM}$ glucose that was restored with $1 \mathrm{mM}$ pyruvate (Additional file 4: Figure S4A-B). Remarkably, HK2-deficient $\mathrm{T}$ cells exhibited an identical response to glucose concentrations as WT $\mathrm{T}$ cells (Additional file 4: Figure S4A-B). Similarly, WT- and HK2-deficient T cells stimulated for 3 days under varying concentrations of glucose showed comparable viability, proliferation as measured by CFSE proliferation index, and proliferation as measured by cell number (Additional file 4: Figure S4C-E). These data demonstrate that $H k 2$ is dispensable in vitro for $\mathrm{T}$ cell viability, activation, and proliferation even under limiting glucose levels.

\section{HK2 deficiency mildly reduces T cell-mediated inflammation in vivo}

It is possible that though we did not see any differences in vitro from loss of $\mathrm{HK} 2$, there could be differences in 

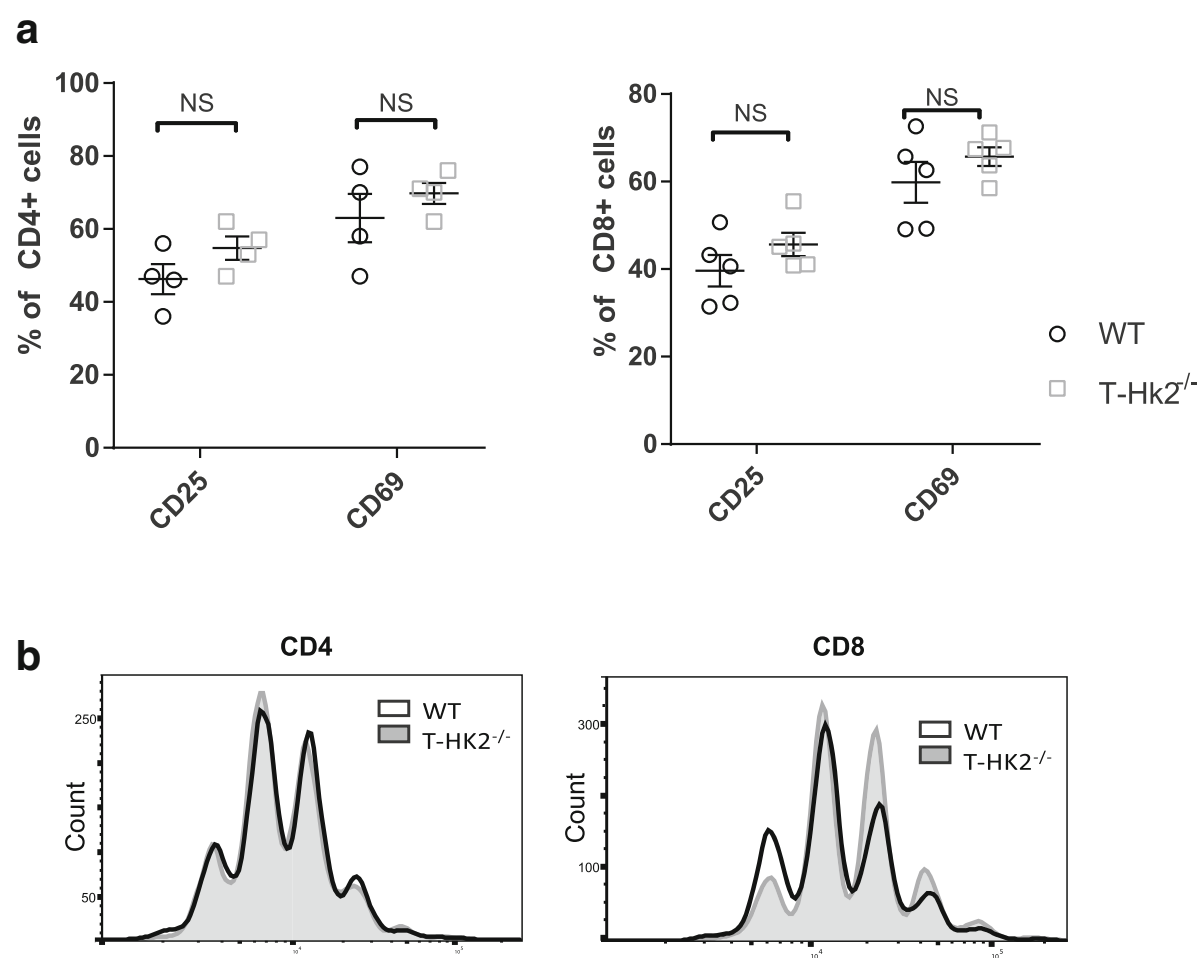

Flourescence (CFSE)

Flourescence (CFSE)

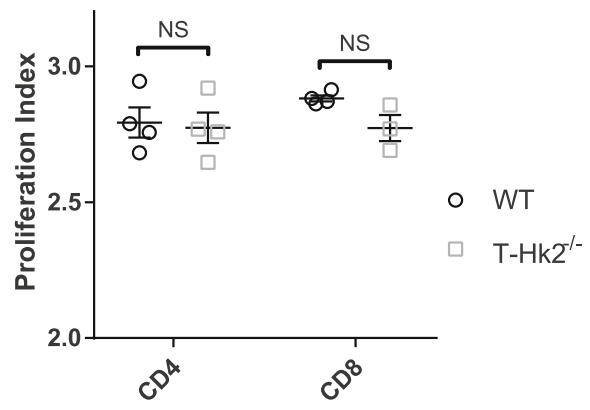

Fig. 2 HK2 is not required for T cell activation and proliferation. Naïve CD4+ and CD8+ T cells were enriched from splenocytes isolated from adult WT and T-Hk2 ${ }^{-/-}$mice. T cells were activated in vitro with anti-CD3/28-coated beads for 24-72 $\mathrm{h}$. a Percent of cells positive for indicated activation markers by flow cytometry gated on CD4+ (left) or CD8+ (right) cells after $24 \mathrm{~h}$ stimulation. Biological replicates, $n=4$ from at least two independent experiments. $\mathbf{b}$ CFSE dilution in activated CD4+ and CD8+T cells after $72 \mathrm{~h}$ of stimulation. Representative image of $n=4$ biological replicates from at least two independent experiments. Quantified by proliferation index on right, $n=3-4$ biological replicates as indicated by individual data points. In all panels unless otherwise noted, error bars represent mean \pm SEM, one-way or two-way ANOVA with Sidak multiple comparisons, ${ }^{*} p<0.05 ;{ }^{*} p<0.01$. NS = not significant

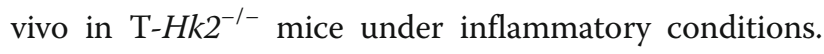
We tested whether HK2 deficiency in T cells would impair $\mathrm{T}$ cell-mediated inflammation by using a mouse model of colitis driven by IL-10 deficiency. $\mathrm{IL}^{10^{-/}}$mice spontaneously develop colitis in a T cell-dependent manner thought to be driven by Th1 and Th17 cytokine-producing cells [30-33]. We generated $H k 2^{\mathrm{fl} / \mathrm{fl}}$, CD4-Cre ${ }^{+}$, and IL- $10^{-/-}$mice that were globally deficient in IL-10 and had a T cell-specific HK2 deficiency (herein referred to as $\mathrm{IL}-10^{-/-}$; $\left.\mathrm{T}-H k 2^{-/-}\right)$. $\mathrm{IL}-10^{-/-}$mice and IL-10 ${ }^{-1-} ; \mathrm{T}-H k 2^{-/-}$mice both developed mild colitis marked by lymphocytic infiltrate in the colon, a failure to gain weight, splenomegaly, and rectal prolapse (Fig. 4a-d). IL-10-sufficient littermate control mice showed no signs of disease. Interestingly, $\mathrm{IL}-10^{-1}$ ${ }^{-}, \mathrm{T}-H k 2^{-/-}$mice developed spontaneous rectal prolapse at a significantly lower rate than $\mathrm{IL}-10^{-/-}$mice, though weight change and splenomegaly were similar between the two (Fig. 4b-d). To further investigate differences in the inflammation between $\mathrm{IL}-10^{-/-}$mice and $\mathrm{IL}-10^{-1}$ ${ }^{-} ; \mathrm{T}-H k 2^{-1-}$ mice, we analyzed tissues by flow cytometry which showed increase cellularity in the mesenteric 


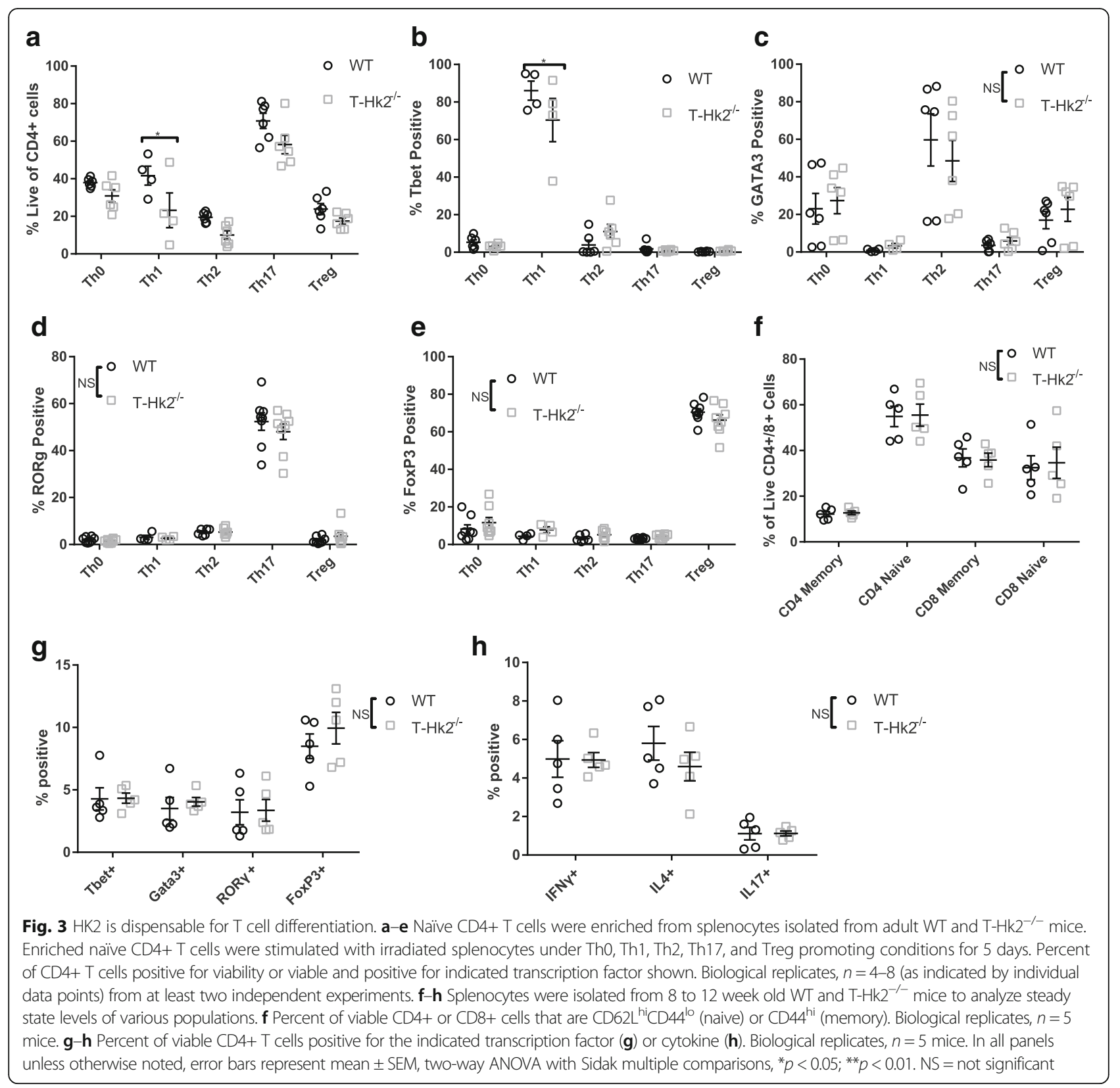

lymph nodes and lamina propria of both $\mathrm{IL} 10^{-/-}$mice and IL-10 $0^{-/-} ; \mathrm{T}-H k 2^{-/-}$when compared to $\mathrm{IL}_{10} 0^{+/+}$mice (Fig. 4e, f). There were no observed differences in CD4 +, CD8+, CD69+, IFN $\gamma+$, IL17+, or FoxP3+ cell numbers in the mesenteric lymph nodes of $\mathrm{IL}^{-10^{-/-}}$mice and $\mathrm{IL}-10^{-/-} ; \mathrm{T}-H \mathrm{k} 2^{-/-}$mice (Fig. 4e, f). However, there was a mild decrease in $\mathrm{CD} 4+\mathrm{T}$ cells in the lamina propria of IL- $10^{-1-}$; T-Hk2 ${ }^{-1-}$ mice (Fig. $4 \mathrm{~g}$ ). Overall, these data indicate that $\mathrm{IL}-10^{-/-} ; \mathrm{T}-H k 2^{-/-}$develop colitis, but with a lower severity than $\mathrm{IL} 10^{-/-}$mice.

We also tested the necessity of HK2 in a mouse model of Th2 lung inflammation by sensitizing WT and $\mathrm{T}-H k^{-/-}$mice with ovalbumin/aluminum hydroxide
(OVA/alum) and provoking airway inflammation subsequently with aerosolized OVA. Inflammation in the airway in response to OVA is known to be a Th2-driven process with a Th17 component [34]. Non-sensitized

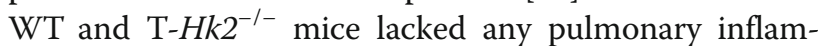
mation after challenge with OVA while both WT and $\mathrm{T}-H k 2^{-/-}$sensitized mice developed significant inflammation (Fig. 5a). Bronchoalveolar lavage after OVA chal-

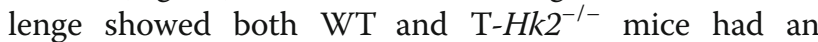
increase in BAL cellularity when compared to mice that were not previously sensitized (Fig. 5b). Absolute levels of $\mathrm{T}$ cells and eosinophils were increased in the BAL of

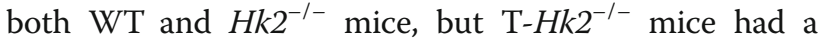




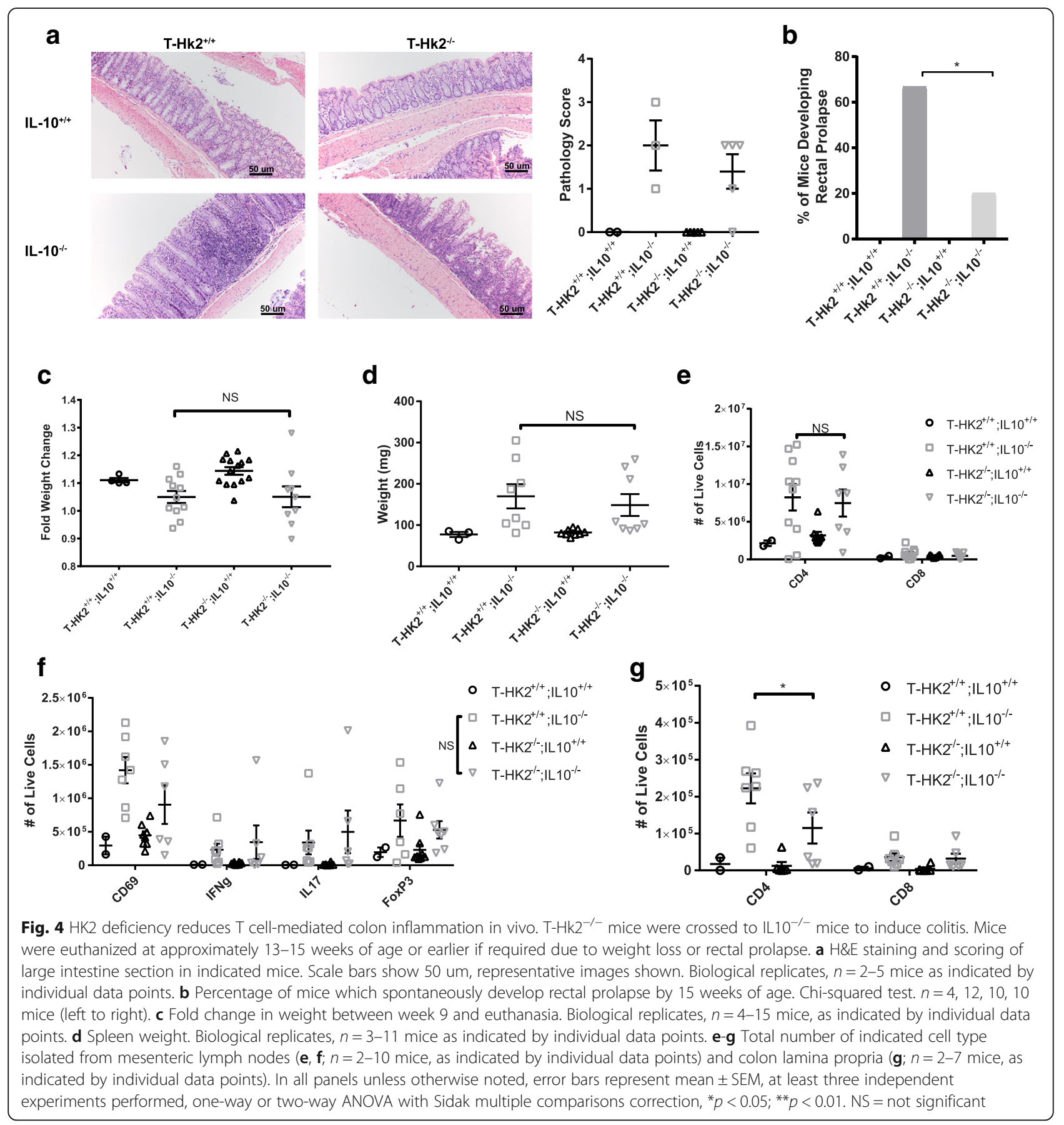

small decrease in eosinophils when compared to WT mice (Fig. 5b). Th2 cytokines in the BAL were increased in both

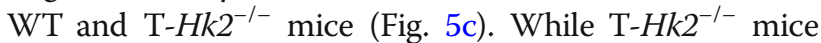
had cytokine levels that trended downward compared to WT mice, the decrease was not statistically significant. Whole lung RNA cytokine levels showed similar results with a significant increase in Th2 and Th17 cytokines in

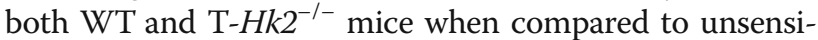
tized mice; IFN $\gamma$, a Th1 cytokine, was not increased
(Fig. 5d-h). Furthermore, similar to BAL levels, RNA levels showed a descending trend in cytokine levels when

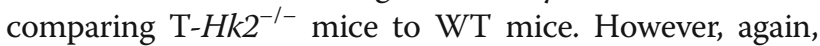
the decrease in cytokine levels of $\mathrm{T}-H k 2^{-1-}$ mice was not statistically significant, though the decrease in IL-17 approached significance $(p=0.06)$. Taken together, these results indicate that $H k 2$ is not strictly required for development of Th2 inflammation, but its presence may modulate the extent of inflammation. 


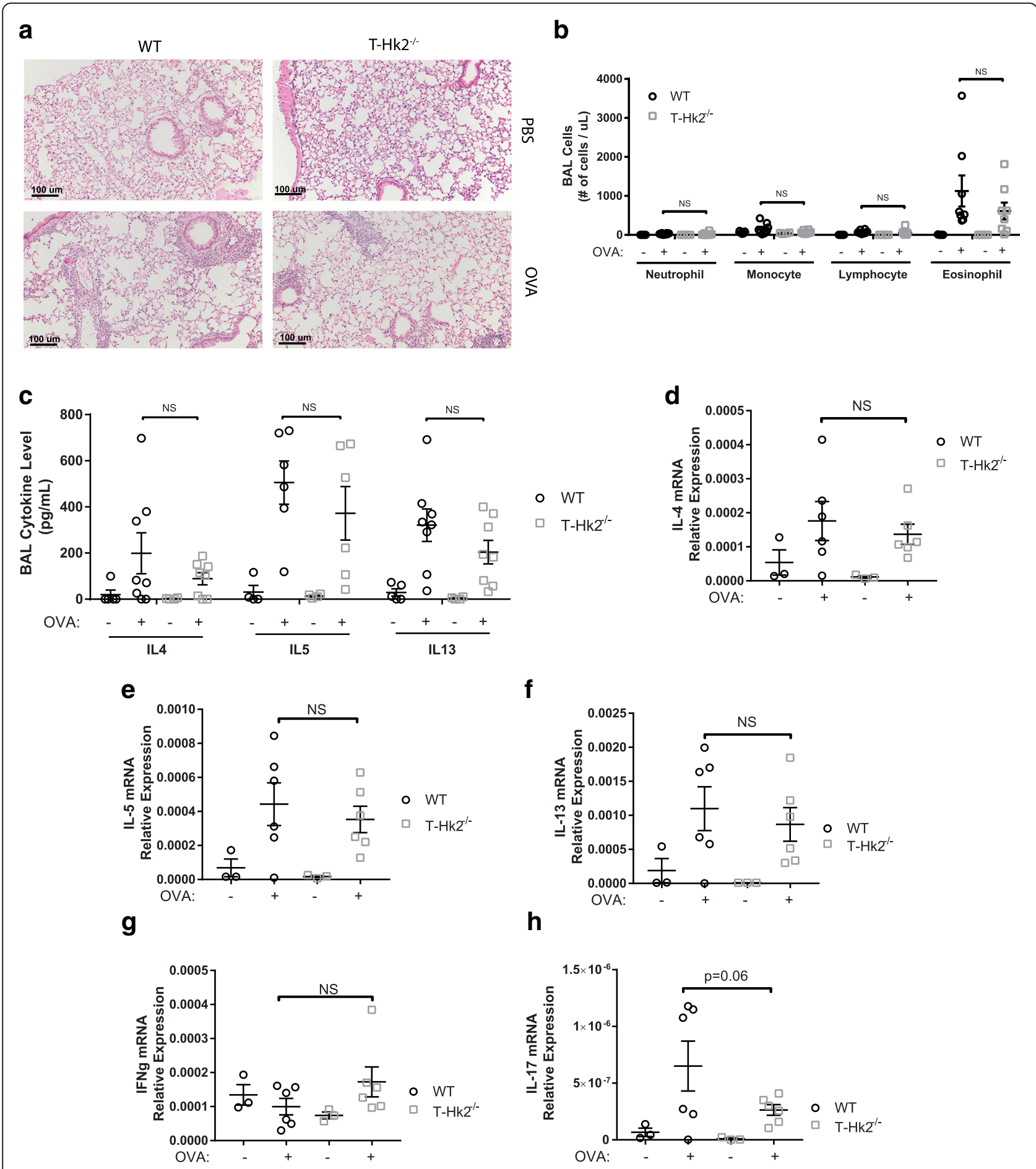

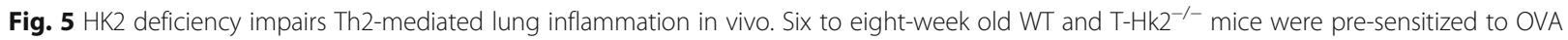
twice, 14 days apart with intraperitoneal injection of OVA/alum or PBS/alum. Twenty-one days after initial pre-sensitization, mice were challenged with aerosolized OVA daily for 3 days. Mice were euthanized for analysis on the fourth day. a H\&E staining of lung tissue. Scale bars show 100 um, representative images shown from $n=4$ mice. $\mathbf{b}$ Number of indicated cells present in BAL fluid (c) ELISA for indicated cytokines in BAL fluid. b, $\mathbf{c}$ Multiple $t$ tests with Holm-Sidak correction for multiple comparisons. $\mathbf{d}-\mathbf{h}$ RNA expression of indicated cytokine relative to $\beta$-actin. In all panels unless otherwise noted, error bars represent mean $\pm \mathrm{SEM}$, biological replicates, $n=5,8,4,8$ mice (left to right) from three independent experiments, unless otherwise noted, one-way ANOVA with Sidak multiple comparisons correction, ${ }^{*} p<0.05 ;{ }^{* *} p<0.01$. NS $=$ not significant 


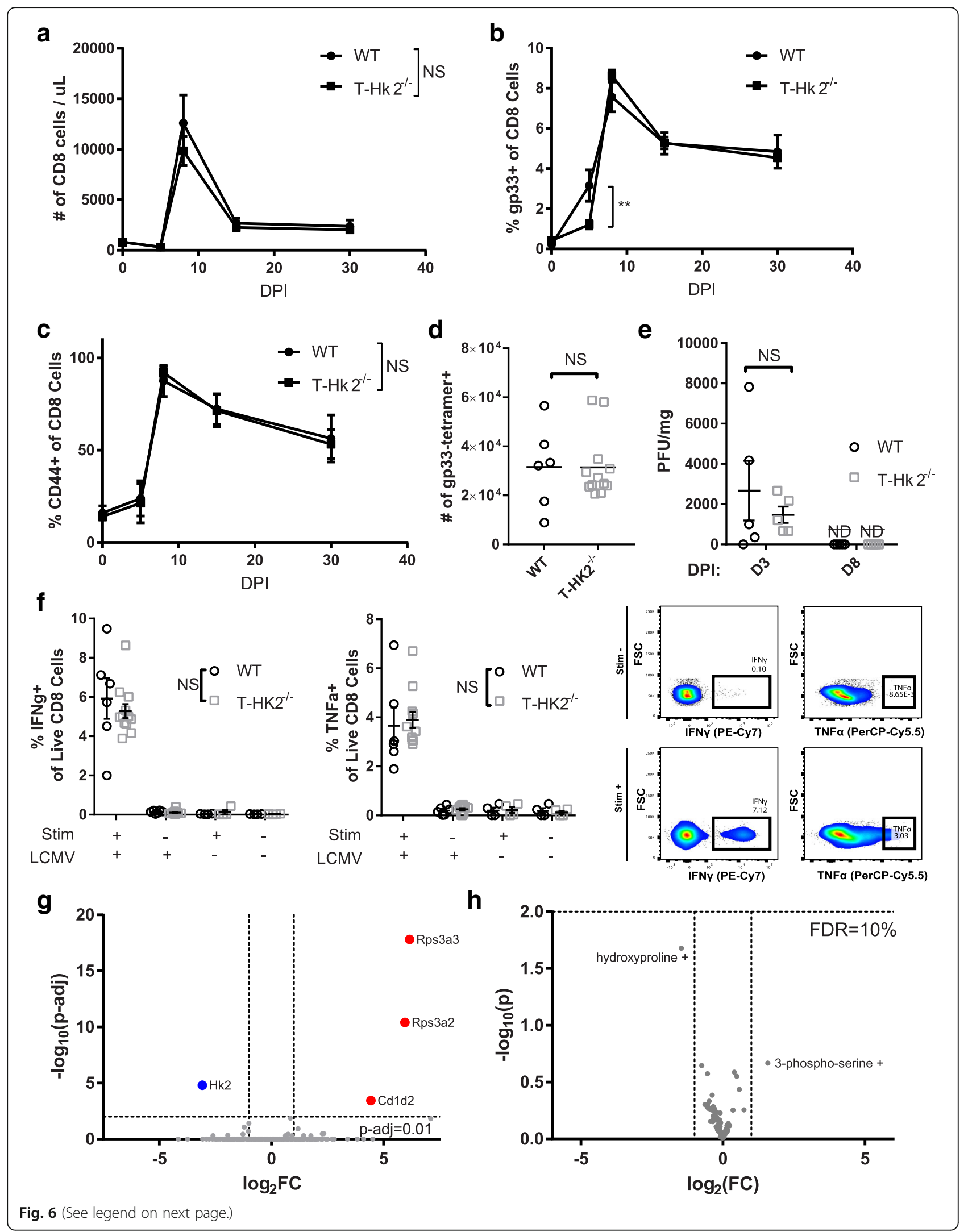




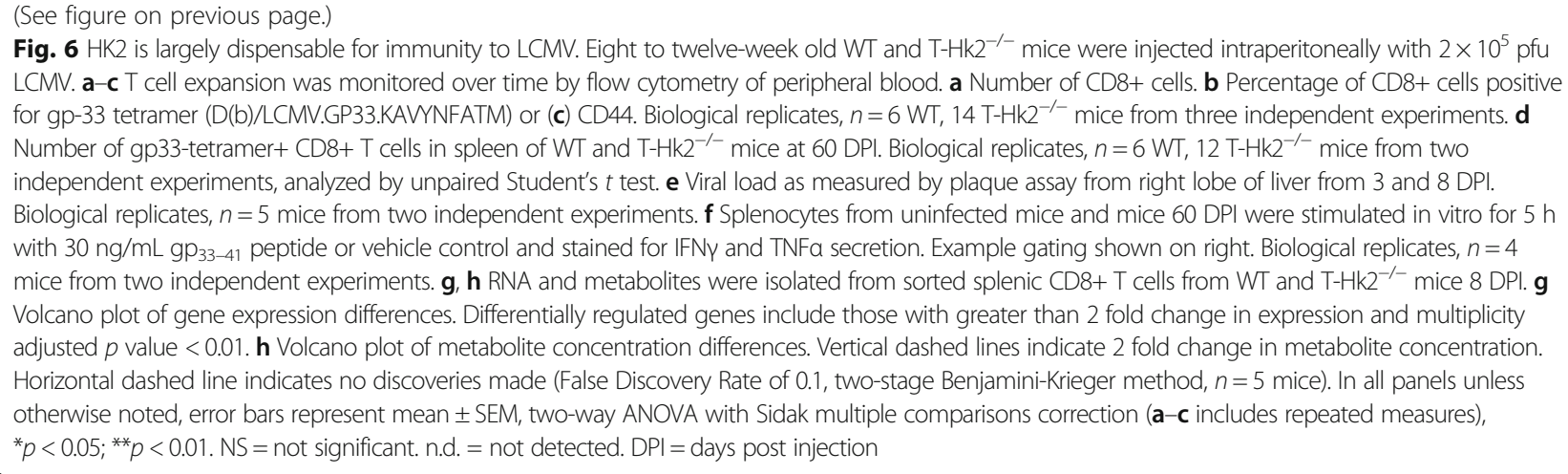

\section{HK2 is not required for T cell-mediated viral immunity}

To test the role of HK2 in viral immunity, we infected $\mathrm{T}-H k 2^{-/-}$mice intraperitoneally with lymphocytic choriomeningitis virus (LCMV). Rapid clearance of the virus after acute infection requires function of CD8+ T cells and maintenance of a LCMV-specific memory population requires CD4+ and CD8+ T cell function $[35,36]$. As we observed a small difference in proliferation of CD8+ T cells in vitro, we hypothesized there could be a defect in the anti-viral proliferative response, but $\mathrm{T}-H k 2^{-/-}$mice show an antigen-specific CD8+ T cell expansion following infection with LCMV identical to that of WT mice; CD8+ T cells expand rapidly and then slowly contract (Fig. 6a). However, the percentage of CD8+ cells specific for the gp33 epitope of LCMV in the blood lags in T- $H k 2^{-1-}$ mice compared to WT mice at $5 \mathrm{DPI}$, though it catches up by 8 DPI and remains at comparable levels for at least 60 days (Fig. 6b, Additional file 3: Figure S3D). Notably, the $\mathrm{T}-H k 2^{-/-}$mice memory population expands and contracts just as the respective population in WT mice (Fig. 6c). Numbers of antigen-specific memory cells in the spleen are also similar between WT and T- $H k 2^{-1-}$ mice at 60 DPI (Fig. 6d) and T-Hk2 $2^{-/-}$mice were able to clear infection by 8 DPI just as WT mice and had a similar viral load at 3 DPI (Fig. 6e). Furthermore, antigen-specific memory cells at $60 \mathrm{DPI}$ are also functional, as they retain their ability to secrete IFN $\gamma$ and TNF $\alpha$ following restimulation with exogenous gp33 peptide (Fig. 6f).

In order to determine if there were any in vivo differences in metabolism between WT and T-Hk2 $2^{-1-}$ mice, we analyzed metabolite and transcript profiles of CD8+ $\mathrm{T}$ cells from the spleens of mice at $8 \mathrm{DPI}$. We found no significant difference in metabolite levels and only four differentially expressed genes between WT and T-Hk2 $2^{-/-}$ mice (Fig. 6g, h). Predictably, of the few genes that were differentially expressed, $H k 2$ stood out as the most downregulated gene in CD8+ $\mathrm{T}$ cells from $\mathrm{T}-H k 2^{-/-}$ mice. Importantly, HK1 was unchanged in both WTand HK2-deficient T cells. These data indicate that HK2 is dispensable for CD8+ T cell-mediated viral immunity.

\section{HK2 is not required for Treg function in vivo}

Although HK2 is not necessary for inflammatory CD4+ and CD8+ lineages, we next determined if HK2 was required for the function of the regulatory $\mathrm{T}$ cell lineage (Treg cells). We generated mice with Treg-specific deletion of the $H k 2$ gene by crossing $H k 2^{\mathrm{f} / \mathrm{fl}}$ mice with FoxP3-YFP-Cre mice. Tregs isolated from these mice (FP3- $H k 2^{-/-}$) did not show appreciable levels of $H k 2$ mRNA transcript (Fig. 7a, Additional file 3: Figure S3E). FP3- $H k 2^{-/-}$mice did not develop any indications of autoimmune disease as would be expected of mice with deficiencies in Treg function [37, 38], as determined by histology, serum ANA titers, weight, and presence of splenomegaly (Fig. 7b-e). Furthermore, they had normal numbers of Tregs in the spleen, as well as normal numbers of naïve and memory CD4 and CD8 T cells (Fig. 7f, g). These data indicate that HK2 is dispensable for Treg function in vivo.

\section{HK2 is not required for normal hematopoiesis}

To determine if there is any defect in the ability of other hematopoietic lineages to differentiate without HK2, we abolished HK2 function in hematopoietic stem cells by crossing Hk2-floxed mice with Vav1-iCre, which expresses Cre in hematopoietic stem cells during development [39]. Resulting $\mathrm{BM}-H \mathrm{~K} 2^{-/-}$mice did not have any significant HK2 expression as determined by western blot (Fig. 8a, Additional file 2: Figure S2B). Furthermore, HK2 deficiency did not impair hematopoiesis as assessed by platelet, white, and red blood cell counts (Fig. 8b). Collectively, these data indicate HK2 is not required for normal hematopoiesis.

\section{Transcriptome differences between $\mathrm{T}$ cells and $\mathrm{T}$ cell leukemia in vivo}

While cancer cells and T cells both upregulated HK2 expression, our data indicate it is only required in the former. For example, HK2 deficiency has been shown to decrease tumor burden in a mouse model of $\mathrm{T}$ cell acute lymphoblastic leukemia (T-ALL) [19]. Finding therapeutics that target neoplastic $\mathrm{T}$ cells while sparing normal $\mathrm{T}$ 


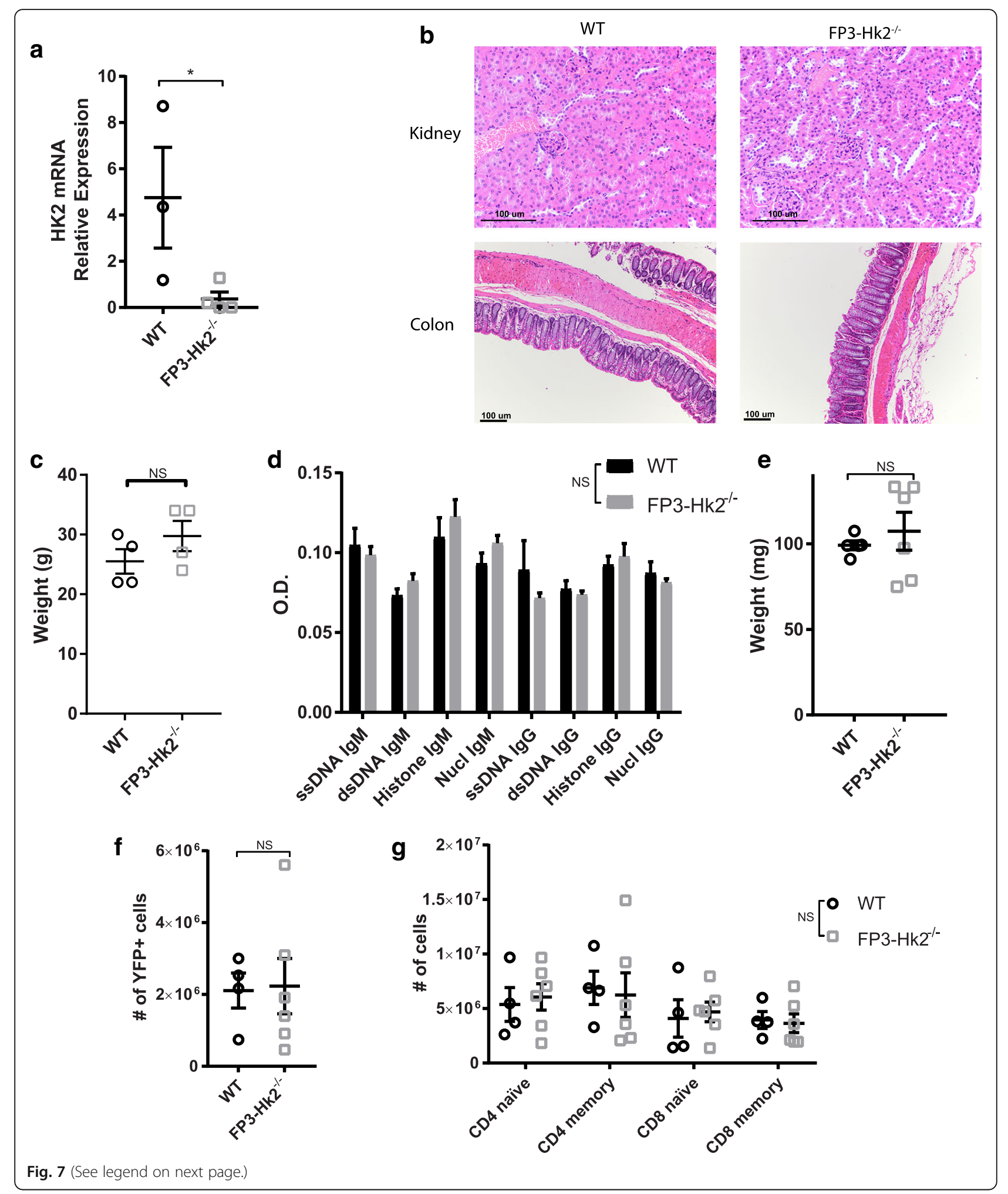


(See figure on previous page.)

Fig. 7 HK2 is not required for Treg function in vivo. Mice with YFP+ HK2-deficient Treg cells were generated (FP3-Hk2 ${ }^{-/-}$mice). a CD4+ cells from spleens of adult WT and FP3-Hk2 $2^{-1-}$ mice were stimulated under Treg promoting conditions and RNA was isolated from sorted YFP+ cells. Hk2 expression relative to $\beta$-actin $\left(n=3 \mathrm{WT}, 4 \mathrm{FP} 3-\mathrm{Hk}^{-/-}\right)$. $\mathbf{b}-\mathbf{g} \mathrm{WT}$ and $\mathrm{FP} 3-\mathrm{Hk} 2^{-/-}$mice were aged to 6 months. $\mathbf{b}$ Representative H\&E stained sections of indicated tissues, $n=4$ mice. Scale bars show 100 um. c Mouse weight; biological replicates, $n=4$ mice. $\mathbf{d}$ Titer of indicated auto-antibodies in serum; biological replicates, $n=4 \mathrm{WT}, 7 \mathrm{FP3}-\mathrm{Hk} 2^{-/-}$mice. e Spleen weight; biological replicates, $n=5 \mathrm{WT}, 6 \mathrm{FP} 3-\mathrm{Hk} 2^{-/-}$mice. $\mathbf{f}$ Number of splenic Treg cells and $\mathbf{g}$ percent of viable CD4+ or CD8+ T cells that are CD62 $\mathrm{L}^{\text {hi }} \mathrm{CD} 44^{\mathrm{lo}}$ (naive) or CD44 ${ }^{\text {hi }}$ (memory); biological replicates, $n=4$ WT, 6 FP3-Hk2 ${ }^{-1-}$ mice. In all panels unless otherwise noted, error bars represent mean \pm SEM, experiments performed twice independently, single-tailed Student's $t$ test or two-way ANOVA with Sidak multiple comparisons correction, ${ }^{*} p<0.05 ;{ }^{* *} p<0.01$. NS $=$ not significant

cells represents an interesting challenge in cancer therapy, as the two share a common origin and common proliferative goals. In order to identify other potential metabolic targets for T-ALL beyond HK2 that would preferentially inhibit leukemia while sparing lymphocytes, we compared in vivo proliferating $\mathrm{T}$ cells and primary mouse T-ALL cells by RNA-seq. WT bone marrow was transduced with retrovirus expressing a constitutively active Notch protein (Notch1-deltaE) and injected into congenic CD45.1 mice. Mice developed CD4 + CD8 + lymphoblasts in their blood and lymphoid organs. WT primary leukemia cells were isolated from the spleens of recipient mice after 6 weeks and compared to WT CD8 $+\mathrm{T}$ cells isolated from mouse spleens 8 days after infection with LCMV (Additional file 3: Figure S3F). A wide range of differentially expressed genes were identified between $\mathrm{T}$ cells and leukemic cells (Fig. 9). Three thousand three hundred thirty-one genes were identified as being significantly differentially expressed between $\mathrm{T}$ cells and leukemia (Fig. 9a), with leukemic samples enriched in expected gene sets such as Myc targets, beta-catenin pathway, and notch1 signaling (Fig. 9b). T cells were unsurprisingly enriched for gene sets involved in $\mathrm{T}$ cell processes such as allograft rejection and IFNY response (Fig. 9b). Of the differentially expressed genes, 167 were metabolic genes in the KEGG database, including 61 upregulated metabolic genes in leukemia (Fig. 9c). Five of the top metabolic genes upregulated in leukemia

a

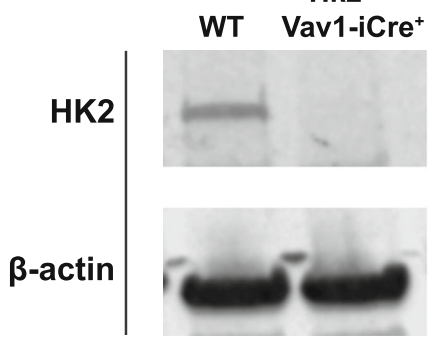

b

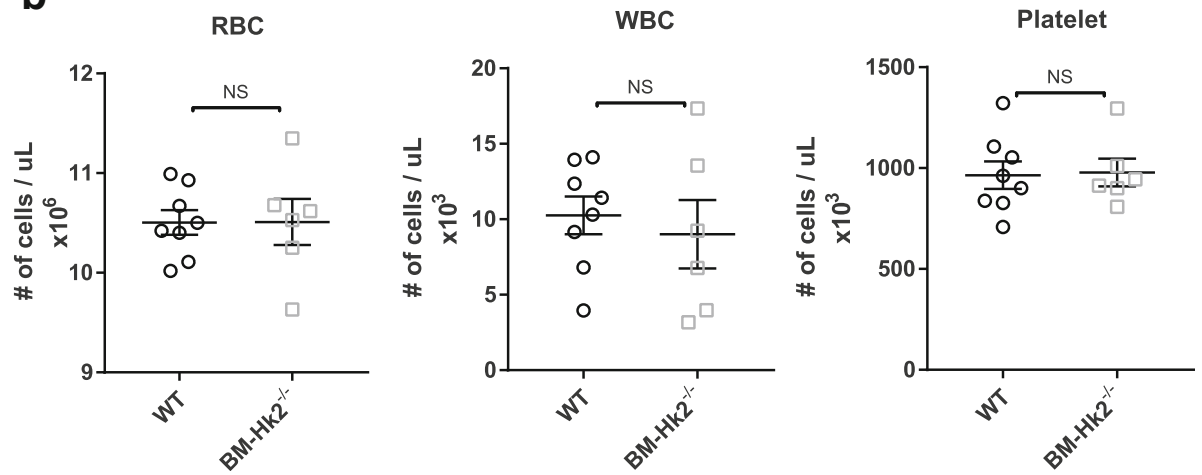

Fig. 8 HK2 is not required for normal hematpoiesis. Mice with HK2-deficient bone marrow were generated $\left(\mathrm{Hk}_{2}{ }^{\mathrm{F} / \mathrm{Fl}} ; \mathrm{Vav} \mathrm{ViCre}^{+}\right.$, referred to as BM$\mathrm{Hk}^{-1-}$ ) a Western blot for protein expression of $\mathrm{HK} 2$ and $\beta$-actin in bone marrow. Representative of four independent experiments $\mathbf{b}$ Peripheral blood counts of indicated hematopoietic lineages in adult WT and $\mathrm{Hk}_{2}{ }^{\mathrm{Fl} / \mathrm{Fl}}$; Vav1-iCre ${ }^{+}$mice. $n=8 \mathrm{WT}, 6 \mathrm{Hk}^{\mathrm{Fl} / \mathrm{Fl}}$; Vav1-iCre ${ }^{+}$mice. In all panels, biological replicates shown, at least two independent experiments performed, error bars represent mean \pm SEM, unpaired $t$ test, ${ }^{*} p<0.05$; ${ }^{* *} p<0.01$. NS = not significant 


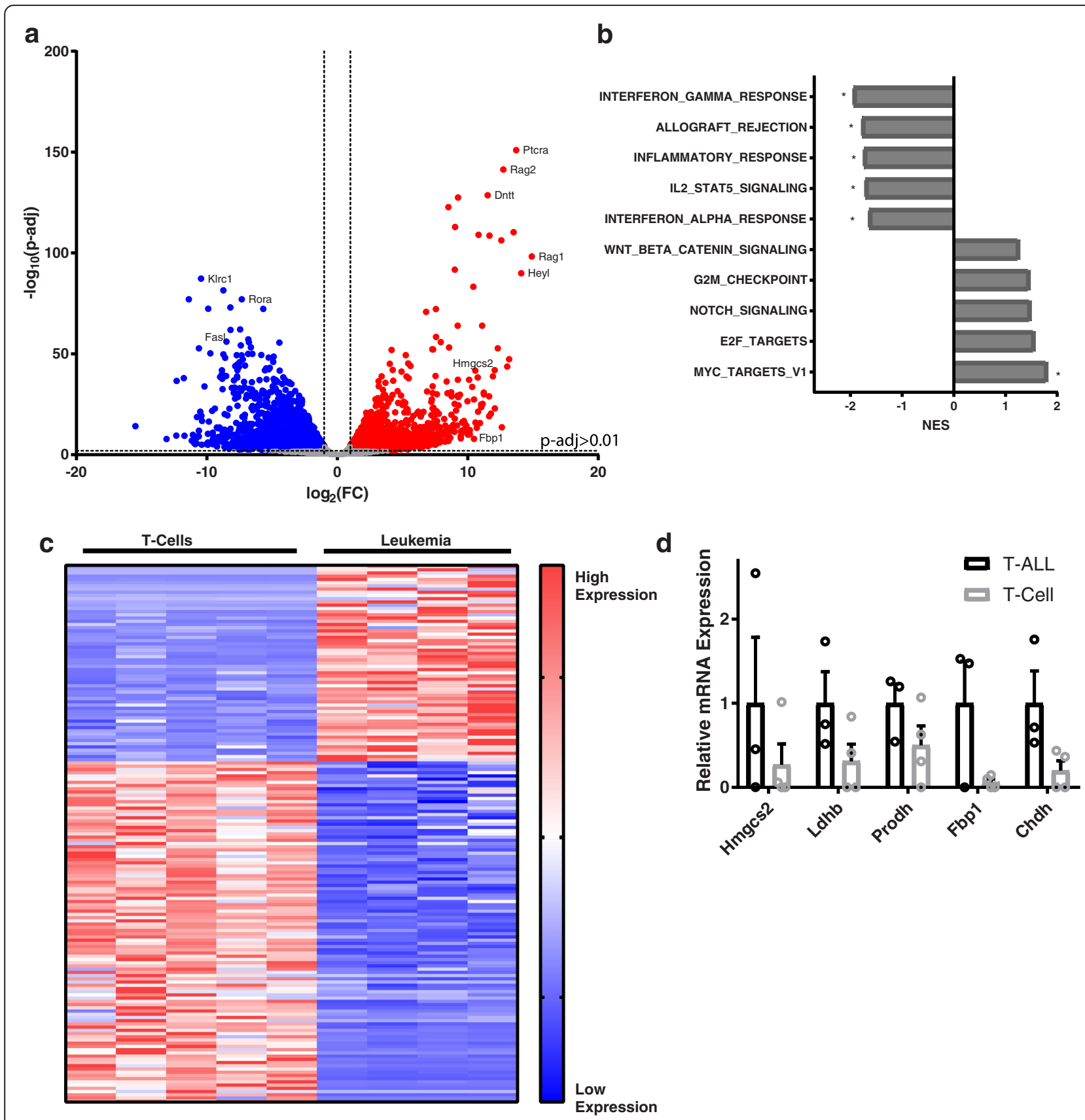

Fig. 9 Transcriptomic and metabolomic analysis reveals essential differences in metabolism of leukemia and lymphocytes. Sorted splenic CD8+ T cells from WT mice 8 days after LCMV infection, compared to sorted T-ALL cells from WT mice 6 weeks after adoptive transfer. RNA isolated from sorted cells and analyzed by RNA-seq a Volcano plot of gene expression differences. Differentially regulated genes include those with greater than 2 fold change in expression and multiplicity adjusted $p$ value $<0.01$. Blue dots are overexpressed in CD8+ T cells, red in T-ALL. b Hallmark pathways enriched in leukemia (positive values) and T cells (negative values) and their normalized enrichment score. Statistical significance shown by * (FWER $p<0.05$ ). c Expression of differentially regulated metabolic genes from the KEGG database. $\mathbf{d}$ Expression of select metabolic genes by RT-PCR relative to $\beta$-actin and Rpl-19. Biological replicates, $n=3-4$ mice, as indicated by individual data points

when compared to CD8+ T cells were Hmgcs2, Chdh, Fbp1, Prodh, and Ldhb. These genes consistently showed higher expression in leukemia cells when re-evaluated by RT-PCR (Fig. 9d). While some differences were statistically significant after $t$ tests, no individual gene reached statistical significance on multiple comparisons correction. Taken together, these data reveal essential differences in $\mathrm{T}$ cell and T-ALL cell metabolism that may be harnessed to selectively inhibit leukemic proliferation. 


\section{Discussion}

In the past decade, there has been a resurgence of targeting metabolism for cancer therapy. Cancer cells undergo metabolic reprogramming to acquire sufficient nutrients to fuel macromolecule synthesis for growth and proliferation $[2,4,12]$. However, the therapeutic window is limited by metabolic rewiring that cancer cells quickly undergo upon inhibition of a particular metabolic pathway thus becoming resistant to therapies targeting metabolism. Normal proliferating $\mathrm{T}$ cells, stem and progenitor cells also display features of metabolic reprogramming similar to cancer cells, further limiting the therapeutic index in targeting metabolism of cancer cells $[1,3,11]$. Moreover, targeting metabolism within $\mathrm{T}$ cells to diminish their proliferation and function could leave patients susceptible to life threatening infections while also rendering useless cutting-edge immunotherapies such as checkpoint blockade and CAR-T cells.

A similar metabolic feature of proliferating cancer cells and $\mathrm{T}$ cells is that they both highly upregulate glycolytic enzymes $[4,10,11,16-18]$. HK2 is the one the most highly upregulated glycolytic enzymes in activated T cells and oncogenic transformed cells [16-18, 20, 21]. Thus, we were surprised with our observation that HK2 is dispensable for the essential function of $\mathrm{T}$ cells-viral immunity. Clearance of acute LCMV infection was completely intact in mice with $H k 2^{-/-} \mathrm{T}$ cells, as was the ability of those mice to develop $\mathrm{T}$ cell memory. Unexpectedly, we found minimal differences in metabolite levels and gene expression between wild-type and HK2-deficient proliferating CD8 $\mathrm{T}$ cells in response to LCMV. Moreover, HK2 deficiency did not impair Treg function. Loss of $\mathrm{HK} 2$ also did not impair normal hematopoiesis indicating normal stem and progenitor cell function. Recent work has also shown that HK2 is required for angiogenesis [40], and this inhibition could theoretically further synergize with cancer cell HK2 inhibition to improve response to therapy. In contrast to the observed dispensability of $\mathrm{HK} 2$ in immunity, we found that HK2 may play a small role in pathological $\mathrm{T}$ cell-mediated inflammation. In a mouse model of colitis, HK2 deficiency conferred a limited degree of protection against inflammation. We speculate that the simplest explanation for this is that pathogenic $\mathrm{T}$ cells have a higher demand for glucose which HK2-deficient cells are unable to meet. Indeed, this effect has been observed in other models of inflammation, and 2-DG has been proposed as a potential therapeutic in autoimmune diseases $[7,8]$. However, it is also possible the microenvironment specific to these models may play a potential role, and it is also possible that $\mathrm{HK} 2$ could have non-metabolic functions contributing to pathogenesis.

It is important to note that recent work from another group supports our finding of $\mathrm{HK} 2$ dispensability in $\mathrm{T}$ cells, but in a model of herpes virus infection [41]. However, while they propose a compensatory upregulation of HK1 - in HK2-deficient T cells, we are unable to confirm that conclusion in our system. There was no observed compensatory increase in HK1 or HK3 expression at the RNA or protein level. Rather, we believe HK2 is dispensable because HK2-mediated glucose phosphorylation only constitutes a small percentage of total glucose phosphorylation in CD4+ and CD8+ T cells.

To identify other targets which could be utilized to selectively inhibit cancer cells, we compared the transcriptome and metabolome of proliferating $\mathrm{T}$ cell leukemia with $\mathrm{T}$ cells proliferating physiologically in response to infection. This analysis revealed striking differences in how the metabolism of a leukemic cell differs from that of a $\mathrm{T}$ cell and identified specific potential targets for cancer therapy, including Hmgcs2, Prodh, Ldhb, Fbp1, and Chdh. This dataset may serve as a significant resource for future studies in cancer therapy to determine if any of the identified highly expressed metabolic genes are also required for leukemia.

\section{Conclusions}

Multiple studies have shown HK2 is essential for cancer growth and development, including in models of breast cancer, lung cancer, and leukemia, leading to interest in HK2 as a potential drug target for cancer therapy. We sought to determine the role of HK2 in T cells, considering the high expression of HK2 in T cells and the potential for HK2 inhibition to adversely affect immunity. We have demonstrated through various animal models that HK2 serves as a mostly redundant enzyme in $\mathrm{T}$ cells. We propose that our findings make HK2 an attractive target for cancer therapy, as it would be expected to have limited immunosuppressive side effects. However, future studies are necessary to further investigate the role HK2 inhibition could play in cancer therapy. Testing HK2 dispensability in the context of other viral infections, other immune cell types, and tumor immunity would be important steps to making more generalizable conclusions.

\section{Additional files}

\footnotetext{
Additional file 1: Figure S1. HK isoform expression in Hk2-/- T cells. CD4+ and CD8+ T cells were enriched from splenocytes isolated from adult WT and T-Hk2-/- mice. T cells were activated in vitro with anti-CD3/28 coated beads for $72 \mathrm{~h}$. HK isoform and $\beta$-actin protein expression in (A) CD4+ and (B) CD8+T cells. Biological replicates, $n=4$. (PDF $5200 \mathrm{~kb}$ )

Additional file 2 Figure S2. Uncropped Gel Images. HK2 and loading control expression in (A) activated CD4+ $T$ cells and (B) bone marrow. (B) Combined exposure shown or (A) individual exposures shown as appropriate. (PDF 2760 kb)

Additional file 3 Figure S3. Example Gating Strategies. (A) Example live CD8 $+T$ cell gating, and subsequent gating for evaluation of activation markers. Similar gating used for CD4+ T cells (B) Gating on single cells for mixed populations of CD4+ and CD8 $+T$ cells followed by gating for
} 
CD62L and CD44 expression (performed on CD4+ and CD8+ T cells separately). (C) Gating on live CD4+ cells for expression of transcription factors. (D) Gating on CD8+ cells for gp-33 tetramer. (E) Gating on CD4+ cells for YFP. (F) Gating on single cells for CD45.2 and GFP expression. (PDF $1300 \mathrm{~kb}$ )

Additional file 4: Figure S4. HK2 is dispensable for T cell function in glucose-limited conditions. Naiive CD4+ and T cells were enriched from splenocytes isolated from adult WT and T-Hk2-/- mice. T cells were activated in vitro with anti-CD3/28 coated beads. (A-B) CD4+ T cells activated in indicated glucose/pyruvate concentrations for $24 \mathrm{~h}$. (B) Percent of CD4+ cells positive for viability and (C) percent of viable CD4+ cells expressing CD69 by flow cytometry. Biological replicates, $n=6$ from 3 independent experiments (C-E) Enriched splenic CD4+ T cells activated for $72 \mathrm{~h}$ in media with $1 \mathrm{mM}$ pyruvate and indicated glucose concentrations. CD4+ T cells analyzed by flow cytometry for (D) percent of cells positive for viability, (E) proliferation as measured by CFSE dilution, and (F) proliferation as measured by cell number. Biological replicates, $n=4$ WT, 5 T-Hk2-l- from 2 independent experiments. ( $F-G)$ Splenocytes were isolated from 8 to 12 week old WT and T-Hk2-/- mice to analyze steady state levels of various populations by indicated marker, $n=5$ mice. $\mathrm{MP}=$ macrophage, $\mathrm{DC}=$ dendritic cell NK = natural killer cell, Neutro = neutrophil. In all panels, error bars represent mean \pm SEM, two-way ANOVA with Sidak multiple comparisons correction, ${ }^{*} p<0.05 ;{ }^{* *} p<0.01$. NS $=$ not significant. (PDF $\left.809 \mathrm{~kb}\right)$

\section{Abbreviations}

2-DG: 2-deoxyglucose; DPI: Days post infection; ECAR: Extracellular acidification rate; FI: Floxed; HK: Hexokinase; LCMV: Lymphocytic choriomeningitis virus; OCR: Oxygen consumption rate; OVA: Ovalbumin; T-ALL: T cell acute lymphoblastic leukemia

\section{Acknowledgements}

We would like to thank the Northwestern University flow cytometry core (RHLCC) and mouse histology core (MHPL). RNA sequencing was performed by the Northwestern High Throughput RNA-Seq Center, within the Division of Pulmonary and Critical Care. We thank Kiwon Nam and Hiam AbdalaValencia for the processing of RNA sequencing samples. The following reagent was obtained through the NIH Tetramer Core Facility: D(b)/ LCMV.GP33.KAVYNFATM. We thank Panagiotis Ntziachristos for his gifts of Notch1-DE plasmid and Platinum-E cells.

\section{Funding}

This work was supported by the NIH (R35CA197532 and 5P01HL071643-13) to N.S.C., NIH (T32 T32HL076139) to S.E.W., NIH (T32 CA009560) to M.M.M. and $101 \mathrm{~B} \times 0007333$ and R01 CA206167 to NH.

\section{Availability of data and materials}

RNA-seq data are available in the Gene Expression Omnibus (GEO) respository, https://www.ncbi.nIm.nih.gov/geo/query/acc.cgi?acc=GSE118326. All other data supporting the manuscript are included within the manuscript or are available from the corresponding author on request.

\section{Authors' contributions}

MMM designed and performed the experiments. NSC, NH, and SEW contributed intellectually to the experimental design and conception. ES contributed to colitis and LCMV experiments. PB and KC helped design and performed asthma experiments. CAM analyzed RNA-seq data. PG conducted LC-MS experiments. HRP designed and supervised serum ANA testing. All authors read and approved the final manuscript.

\section{Ethics approval and consent to participate}

All animals were housed and bred in the Northwestern animal vivarium and procedures were approved by Institutional Animal Care and Use Committee (IACUC) at Northwestern University.

\section{Consent for publication}

Not applicable.

\section{Competing interests}

The authors declare that they have no competing interests.

\section{Publisher's Note}

Springer Nature remains neutral with regard to jurisdictional claims in published maps and institutional affiliations.

\section{Author details}

'Department of Medicine, Northwestern University Feinberg School of Medicine, McGaw Pavilion, Rm. M-334, 240 East Huron Street, Chicago, IL 60611, USA. ${ }^{2}$ Department of Biochemistry and Molecular Genetics, Northwestern University Feinberg School of Medicine, Chicago, IL 60611, USA. ${ }^{3}$ Metabolomics Core Facility, Northwestern University Robert H. Lurie Comprehensive Cancer Center, Chicago, IL 60611, USA. ${ }^{4}$ Department of Biochemistry and Molecular Genetics, University of Illinois at Chicago, Chicago, IL 60607, USA.

Received: 13 July 2018 Accepted: 1 August 2018

Published online: 17 August 2018

\section{References}

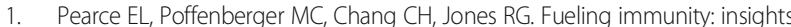
into metabolism and lymphocyte function. Science. 2013;342(6155):1242454.

2. DeBerardinis RJ, Chandel NS. Fundamentals of cancer metabolism. Sci Adv 2016;2(5):e1600200.

3. Andrejeva G, Rathmell JC. Similarities and distinctions of cancer and immune metabolism in inflammation and tumors. Cell Metab. 2017;26(1):49-70.

4. Lunt SY, Vander Heiden MG. Aerobic glycolysis: meeting the metabolic requirements of cell proliferation. Annu Rev Cell Dev Biol. 2011;27:441-64.

5. Chang $\mathrm{CH}$, Curtis JD, Maggi LB Jr, Faubert B, Villarino AV, O'Sullivan D, Huang SC, van der Windt GJ, Blagih J, Qiu J, et al. Posttranscriptional control of T cell effector function by aerobic glycolysis. Cell. 2013;153(6):1239-51.

6. Blagih J, Coulombe F, Vincent EE, Dupuy F, Galicia-Vazquez G, Yurchenko E, Raissi TC, van der Windt GJ, Viollet B, Pearce EL, et al. The energy sensor AMPK regulates T cell metabolic adaptation and effector responses in vivo. Immunity. 2015:42(1):41-54.

7. Yin Y, Choi SC, Xu Z, Perry DJ, Seay H, Croker BP, Sobel ES, Brusko TM, Morel L. Normalization of CD4+ T cell metabolism reverses lupus. Sci Transl Med. 2015;7(274):274ra218.

8. Yang Z, Fujii H, Mohan SV, Goronzy JJ, Weyand CM. Phosphofructokinase deficiency impairs ATP generation, autophagy, and redox balance in rheumatoid arthritis T cells. J Exp Med. 2013;210(10):2119-34.

9. Wang T, Marquardt C, Foker J. Aerobic glycolysis during lymphocyte proliferation. Nature. 1976;261(5562):702-5.

10. Michalek RD, Gerriets VA, Jacobs SR, Macintyre AN, Maclver NJ, Mason EF, Sullivan SA, Nichols AG, Rathmell JC. Cutting edge: distinct glycolytic and lipid oxidative metabolic programs are essential for effector and regulatory CD4+ T cell subsets. J Immunol. 2011;186(6):3299-303.

11. Gerriets VA, Rathmell JC. Metabolic pathways in T cell fate and function. Trends Immunol. 2012:33(4):168-73.

12. Shaw RJ. Glucose metabolism and cancer. Curr Opin Cell Biol. 2006;18(6): 598-608.

13. Chang CH, Qiu J, O'Sullivan D, Buck MD, Noguchi T, Curtis JD, Chen Q, Gindin M, Gubin MM, van der Windt GJ, et al. Metabolic competition in the tumor microenvironment is a driver of cancer progression. Cell. 2015;162(6):1229-41.

14. Ho PC, Bihuniak JD, Macintyre AN, Staron M, Liu X, Amezquita R, Tsui YC, Cui G, Micevic G, Perales JC, et al. Phosphoenolpyruvate is a metabolic checkpoint of anti-tumor T cell responses. Cell. 2015;162(6):1217-28.

15. Wilson JE. Isozymes of mammalian hexokinase: structure, subcellular localization and metabolic function. J Exp Biol. 2003;206(Pt 12):2049-57.

16. Patra KC, Wang Q, Bhaskar PT, Miller L, Wang Z, Wheaton W, Chandel N, Laakso M, Muller WJ, Allen EL, et al. Hexokinase 2 is required for tumor initiation and maintenance and its systemic deletion is therapeutic in mouse models of cancer. Cancer Cell. 2013;24(2):213-28.

17. Botzer LE, Maman S, Sagi-Assif O, Meshel T, Nevo I, Yron I, Witz IP. Hexokinase 2 is a determinant of neuroblastoma metastasis. Br J Cancer. 2016;114(7):759-66.

18. Wang L, Xiong H, Wu F, Zhang Y, Wang J, Zhao L, Guo X, Chang LJ, Zhang Y, You MJ, et al. Hexokinase 2-mediated Warburg effect is required for PTEN- and p53-deficiency-driven prostate cancer growth. Cell Rep. 2014;8(5):1461-74.

19. Kishton RJ, Barnes CE, Nichols AG, Cohen S, Gerriets VA, Siska PJ, Macintyre AN, Goraksha-Hicks P, de Cubas AA, Liu T, et al. AMPK is essential to balance glycolysis and mitochondrial metabolism to control T-ALL cell stress and survival. Cell Metab. 2016;23(4):649-62. 
20. Shi LZ, Wang R, Huang G, Vogel P, Neale G, Green DR, Chi H. HIF1alphadependent glycolytic pathway orchestrates a metabolic checkpoint for the differentiation of TH17 and Treg cells. J Exp Med. 2011;208(7):1367-76.

21. Tan H, Yang K, Li Y, Shaw TI, Wang Y, Blanco DB, Wang X, Cho JH, Wang H, Rankin $\mathrm{S}$, et al. Integrative proteomics and phosphoproteomics profiling reveals dynamic signaling networks and bioenergetics pathways underlying T cell activation. Immunity. 2017;46(3):488-503.

22. Dang EV, Barbi J, Yang HY, Jinasena D, Yu H, Zheng Y, Bordman Z, Fu J, Kim $Y$, Yen HR, et al. Control of $T(H) 17 / T($ reg) balance by hypoxia-inducible factor 1. Cell. 2011;146(5):772-84.

23. Sukumar M, Liu J, Ji Y, Subramanian M, Crompton JG, Yu Z, Roychoudhuri R, Palmer DC, Muranski P, Karoly ED, et al. Inhibiting glycolytic metabolism enhances CD8+ T cell memory and antitumor function. J Clin Invest. 2013; 123(10):4479-88.

24. Sena LA, Li S, Jairaman A, Prakriya M, Ezponda T, Hildeman DA, Wang CR, Schumacker PT, Licht JD, Perlman $\mathrm{H}$, et al. Mitochondria are required for antigen-specific $\mathrm{T}$ cell activation through reactive oxygen species signaling. Immunity. 2013;38(2):225-36.

25. Steinert EM, Schenkel JM, Fraser KA, Beura LK, Manlove LS, Igyarto BZ, Southern PJ, Masopust D. Quantifying memory CD8 T cells reveals regionalization of immunosurveillance. Cell. 2015;161(4):737-49.

26. Erben U, Loddenkemper C, Doerfel K, Spieckermann S, Haller D, Heimesaat MM, Zeitz M, Siegmund B, Kuhl AA. A guide to histomorphological evaluation of intestinal inflammation in mouse models. Int J Clin Exp Pathol. 2014;7(8):4557-76.

27. Bryce PJ, Mathias CB, Harrison KL, Watanabe T, Geha RS, Oettgen HC. The H1 histamine receptor regulates allergic lung responses. J Clin Invest. 2006; 116(6):1624-32.

28. Welsh RM, Seedhom MO. Lymphocytic choriomeningitis virus (LCMV): propagation, quantitation, and storage. Curr Protoc Microbiol. 2008;Chapter 15:Unit 15A. 11

29. Jacobs SR, Herman CE, Maclver NJ, Wofford JA, Wieman HL, Hammen JJ, Rathmell JC. Glucose uptake is limiting in T cell activation and requires CD28-mediated Akt-dependent and independent pathways. J Immunol. 2008;180(7):4476-86

30. Berg DJ, Davidson N, Kuhn R, Muller W, Menon S, Holland G, ThompsonSnipes L, Leach MW, Rennick D. Enterocolitis and colon cancer in interleukin-10-deficient mice are associated with aberrant cytokine production and CD4(+) TH1-like responses. J Clin Invest. 1996;98(4):1010-20.

31. Chichlowski M, Sharp JM, Vanderford DA, Myles MH, Hale LP. Helicobacter typhlonius and Helicobacter rodentium differentially affect the severity of colon inflammation and inflammation-associated neoplasia in IL10-deficient mice. Comp Med. 2008;58(6):534-41.

32. Hoshi N, Schenten D, Nish SA, Walther Z, Gagliani N, Flavell RA, Reizis B, Shen Z, Fox JG, Iwasaki A, et al. MyD88 signalling in colonic mononuclear phagocytes drives colitis in IL-10-deficient mice. Nat Commun. 2012;3:1120.

33. Kuhn R, Lohler J, Rennick D, Rajewsky K, Muller W. Interleukin-10-deficient mice develop chronic enterocolitis. Cell. 1993;75(2):263-74.

34. Afshar R, Medoff BD, Luster AD. Allergic asthma: a tale of many T cells. Clin Exp Allergy. 2008;38(12):1847-57.

35. Shedlock DJ, Shen H. Requirement for CD4 T cell help in generating functional CD8 T cell memory. Science. 2003;300(5617):337-9.

36. Fung-Leung WP, Kundig TM, Zinkernagel RM, Mak TW. Immune response against lymphocytic choriomeningitis virus infection in mice without CD8 expression. J Exp Med. 1991;174(6):1425-9.

37. Zheng Y, Rudensky AY. Foxp3 in control of the regulatory $T$ cell lineage. Nat Immunol. 2007:8(5):457-62.

38. Shrestha S, Yang K, Guy C, Vogel P, Neale G, Chi H. Treg cells require the phosphatase PTEN to restrain TH1 and TFH cell responses. Nat Immunol. 2015;16(2):178-87.

39. de Boer J, Williams A, Skavdis G, Harker N, Coles M, Tolaini M, Norton T, Williams K, Roderick K, Potocnik AJ, et al. Transgenic mice with hematopoietic and lymphoid specific expression of Cre. Eur J Immunol. 2003;33(2):314-25.

40. Yu P, Wilhelm K, Dubrac A, Tung JK, Alves TC, Fang JS, Xie Y, Zhu J, Chen Z, De Smet F, et al. FGF-dependent metabolic control of vascular development. Nature. 2017;545(7653):224-8

41. Varanasi SK, Jaggi U, Hay N, Rouse BT. Hexokinase II may be dispensable for CD4 T cell responses against a virus infection. PLoS One. 2018;13(1): e0191533.

Ready to submit your research? Choose BMC and benefit from:

- fast, convenient online submission

- thorough peer review by experienced researchers in your field

- rapid publication on acceptance

- support for research data, including large and complex data types

- gold Open Access which fosters wider collaboration and increased citations

- maximum visibility for your research: over $100 \mathrm{M}$ website views per year

At BMC, research is always in progress.

Learn more biomedcentral.com/submissions 